\title{
Einführung in das Schwerpunktthema
}

\section{Gesellschaftlicher Umgang mit Risiken}

\section{Von Ulrich Petschow und Jesko Hirschfeld}

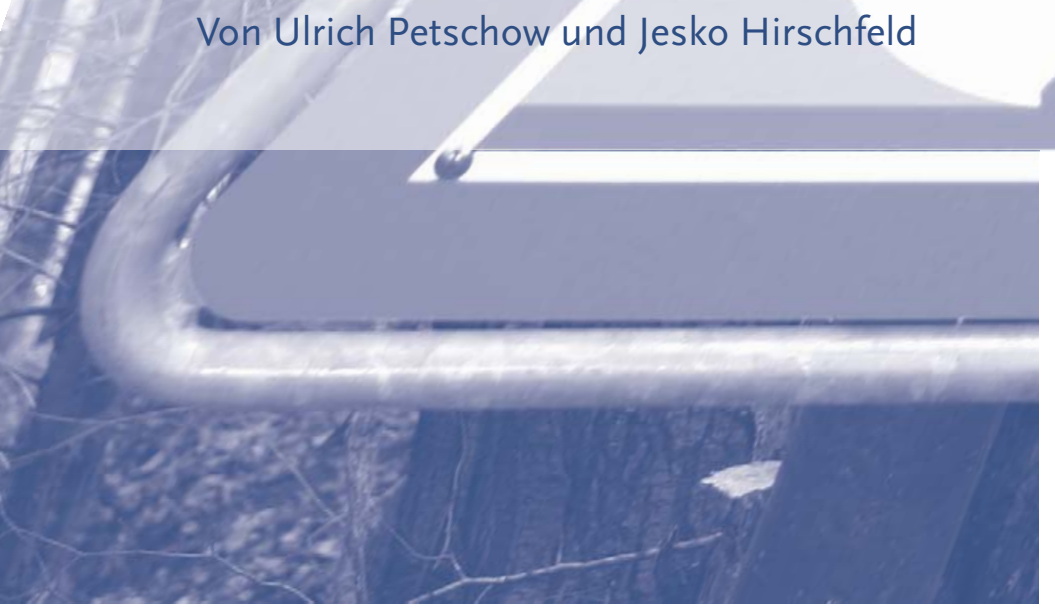

2 005: Das verheerende Erdbeben von Lissabon jährte sich 2 gerade zum 250sten Mal, begleitet von internationalem Gedenken. Es ist keineswegs die einzige historische Katastrophe, die sich im kollektiven Katastrophengedächtnis festgesetzt hat, aber an diesem Ereignis lassen sich die Diskurse der Moderne nachzeichnen.

Zwei Linien sind dabei zu typisieren: Der Wille Gottes oder die Unvernunft des Menschen. Zivilisationskritische Zeitgenossen wie Rousseau merkten an, „dass nicht die Natur zwanzigtausend Häuser von sechs bis sieben Stockwerken zusammengebaut hatte und dass, wenn die Einwohner der Stadt gleichmäßiger verstreut und leichter beherbergt gewesen wären,...die Verheerung weit geringer ... und vielleicht gar nicht geschehen wäre“. Diese Vorstellung verweist darauf, dass „Naturkatastrophen“ (der Wille Gottes) eben keine Natur- sondern vielmehr Kulturkatastrophen darstellen, eine Erfahrung, die bis heute die Wahrnehmung nicht wirklich erfasst hat.

Der Schwerpunkt beleuchtet den gesellschaftlichen Umgang mit Risiken in durchaus heterogenen Kontexten. Risiken entstehen durch die Entwicklung von Technologien, wirtschaftlichem Handeln und dem Umgang mit der Natur. Die Vielfalt der Risiken entspricht der Vielfalt menschlichen Handelns. Dabei gehen wir das Wagnis (oder Risiko?) ein, in dem hier zur Verfügung stehenden Raum dieses komplexe Thema nur ansatzweise ausloten zu können.

\section{Die Beiträge im Einzelnen}

Der Beitrag von Arnim von Gleich orientiert auf Innovationen und das damit zwangsläufig verbundene Nichtwissen. Er fordert, Innovationen grundsätzlich dem Vorsorgeprinzip zu unterwerfen - bei hoher Eingriffstiefe und Wirkmächtigkeit auch dann, wenn noch keinerlei konkrete Gefährdungspotenziale identifiziert sind.
Stefan Böschen setzt sich kritisch mit der Institutionalisierung des gesundheitlichen Verbraucherschutzes in Folge der BSE-Krise auseinander. Er fragt, ob wir die richtigen Schlüsse gezogen und Vorkehrungen getroffen haben.

Franz Prettenthaler analysiert auf Grundlage eines internationalen Vergleichs die Defizite aktuell genutzter Transfermechanismen für Hochwasserrisiken und macht konkrete Reformvorschläge. Er plädiert für eine Einschränkung des Wettbewerbs und der Wahlfreiheit der Versicherten.

Michael Huber erörtert die aktuellen Schwierigkeiten bei der Aufrechterhaltung des britischen „Gentlemen's Agreements“ zwischen Staat und Hochwasserversicherungen angesichts steigender Hochwasserhäufigkeiten und wachsender Schadenspotenziale.

Wolf R. Dombrowsky identifiziert die Illusionen, Irrtümer und Exkulpierungsmechanismen heutiger Risikopolitik und plädiert für das offene Aushandeln eines sozialen Kontraktes zur Handhabung von Nachteilen in einer solidarischen Wagnisgemeinschaft.

Das IÖW bearbeitet zurzeit zwei Projekte zum Umgang mit Hochwasserrisiken: „Kosten-Nutzen-Analyse von Hochwasserschutzmaßnahmen“ für das Umweltbundesamt und „Integrative Nutzung des technischen Hochwasserrückhalts in Poldern und Talsperren “ im Rahmen eines vom BMBF geförderten Verbundprojektes an der Unstrut. Dabei wird deutlich, dass die gesellschaftlichen Institutionen weiterhin große Schwierigkeiten haben, staatliche Regelungsräume mit naturräumlichen Problemen kompatibel zu machen.

Kurzfristige politische Opportunitäten und inadäquate ökonomische Anreizsysteme stehen einer wirksamen Risikovorsorge und einer Reduzierung des Schadenspotenzials im Wege. Und schließlich führt der mangelnde Einbezug überregionaler, langfristiger, indirekter und schwierig monetarisierbarer Wirkungen zu einer systematischen Überbewertung des technischen Hochwasserschutzes gegenüber nachhaltigen und zugleich risikominimierenden Flächennutzungsstrategien.

\section{AUTOREN + KONTAKT}

Ulrich Petschow ist Leiter, Jesko Hirschfeld wissenschaftlicher Mitarbeiter des Forschungsfeldes Umweltökonomie und Umweltpolitik am Institut für ökologische Wirtschaftsforschung (IÖW). IÖW, Potsdamer Str. 105, D-10785 Berlin. Tel.: 030/884594-0, E-Mail: ulrich.petschow@ioew.de, jesko.hirschfeld@ioew.de
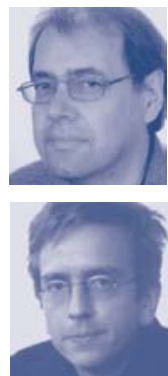
(c) 20I0 Authors; licensee IÖW and oekom verlag. This is an article distributed under the terms of the Creative Commons Attribution Non-Commercial No Derivates License (http://creativecommons.org/licenses/by-nc-nd/3.o/), which permits unrestricted use, distribution, and reproduction in any medium, provided the original work is properly cited. 